\title{
Tecnura
}

\section{Regionalización de ecuaciones para el cálculo de curvas de intensidad, duración y frecuencia mediante mapas de isolíneas en el departamento de Boyacá}

\author{
Regionalization of equations to calculate curves of intensity, duration and \\ frequency using isoline maps in the department of Boyacá
}

\author{
Jorge Enrique Muñoz Barragán', Eduardo Zamudio Huertas²
}

Fecha de recepción: 16 de abril de 2018

Fecha de aceptación: 30 de agosto de 2018

Cómo citar: Muñoz B., J.E y Zamudio H., E. (2018). Regionalización de ecuaciones para el cálculo de curvas de intensidad, duración y frecuencia mediante mapas de isolíneas en el departamento de Boyacá. Tecnura, 22(58), 53-64. DOI: https://doi.org/10.14483/22487638.14295

\section{Resumen}

Contexto: Para el diseño de la mayoría de obras civiles resulta necesario contemplar el factor hidrológico dentro de los estudios o diseños correspondientes al proyecto. En el caso de Colombia, se cuenta con una amplia red hidrometeorológica, administrada por distintas entidades que brindan una completa cobertura del país para el registro y cuantificación de los fenómenos hidrológicos. No obstante, la información correspondiente no se encuentra procesada, como en el caso particular de las curvas de Intensidad, Duración y Frecuencia (IDF) y por esto cuando no se dispone de dichas curvas IDF, se recurre a métodos alternativos poco precisos para su estimación, o sencillamente se utiliza una ecuación aplicable a otras regiones cercanas, por lo cual se desarrollan cálculos poco acertados, dadas las diferencias meteorológicas entre regiones.

Método: Con el fin de dar una solución favorable al problema planteado, se desarrolla la investigación expuesta en este artículo, desarrollando un modelo práctico y preciso que permita definir la ecuación que relacione las variables IDF para cualquier punto dado dentro del área de estudio. Esta investigación considera el departamento de Boyacá, aprovechando sus cualidades geográficas y meteorológicas, que lo hacen la zona más adecuada para adelantar el estudio. No obstante, lo desarrollado en esta publicación es aplicable y puede repetirse para las demás regiones del país, como se espera, mediante otros proyectos.

Resultados: El método de las isolíneas fue el modelo aplicado para la regionalización de las curvas IDF del departamento de Boyacá, gracias a la bondad de los resultados presentados en su aplicación y a la facilidad en su uso final. Se encuentra que existe una relación entre los parámetros que describen una ecuación IDF y su ubicación geoespacial, por lo cual posible regionalizar curvas IDF mediante el uso de mapas de isolíneas cuyo desarrollo se presenta en este artículo. Mediante prueba de validación cruzada se encontró que el error, entre un valor real convencionalmente calculado, y un valor extraído de los mapas de regionalización, se encuentra alrededor del 10\%.

Conclusiones: Se demostró la efectiva aplicabilidad del método de isolíneas, en la regionalización de

1 Ingeniero civil, especialista en Recursos Hídricos. Docente Universidad Santo Tomás sede Villavicencio. Villavicencio, Colombia. Contacto: jorgemunozb@usantotomas.edu.co

2 Ingeniero civil, especialista en Estadística, magíster en Recursos Hidráulicos. Docente de la Universidad Distrital Francisco José de Caldas. Bogotá, Colombia. Contacto: ezamudioh@udistrital.edu.co 
curvas IDF, con un alto nivel de certeza. Con los resultados se garantiza la posibilidad de determinar las constantes que describen la ecuación de las relaciones IDF, para cualquier punto del territorio del departamento de Boyacá. Este método, además de garantizar una buena confiabilidad, se distingue por ser bastante sencillo en su aplicación de manera independiente a variables diferentes a la ubicación geoespacial del punto de análisis. Palabras clave: precipitación, regionalización, Iluvia, intensidad, duración, frecuencia, isolíneas, pluviógrafo, pluviómetro.

\section{Abstract}

Context: For the design of civil works, it is necessary to contemplate the hydrological factor within the studies or designs corresponding to the project. In particular, Colombia has an extensive hydrometeorological network, managed by different entities that provide a complete coverage of the country for the registration and quantification of hydrological phenomena; however, the corresponding information is not processed (for example, Intensity, Duration, and Frequency curves [IDF]). When this information is not available, inaccurate methods are used for its their estimation, or an equation applicable to other nearby regions is used; hence, inaccurate calculations are developed given the meteorological differences between regions.

Method: In order to give a favorable solution to the problem posed, the research presented in this article develops a practical and precise model that allows to define the equation that relates the IDF variables for any given point within the study area. This investigation considers the department of Boyacá, taking advantage of its geographical and meteorological qualities, which make it the most suitable area to carry out the study. However, what is developed in this publication is applicable and can be repeated for the other regions of the country, as expected, through other projects.

Results: The isoline method was applied for the regionalization of the IDF curves of the department of Boyacá, due to the precision of the results and its ease of use. It is found that there is a relationship between the parameters that describe an IDF equation and its geospatial location, so it is possible to regionalize IDF curves by using isoline maps (the development is presented in this article). By means of a cross validation test, it was found that the error between a real value conventionally calculated and a value extracted from the regionalization maps is around 10\%. Conclusions: The effective applicability of the isolines method in the regionalization of IDF curves with a high level of certainty was demonstrated. With the results, the possibility of determining the constants that describe the equation of the IDF relationships for any point of the territory of the department of Boyacá is guaranteed. This method, in addition to guaranteeing good reliability, is distinguished by being quite simple in its application independently to variables other than the geospatial location of the analysis point.

Keywords: precipitation, regionalization, rain, intensity, duration, frequency, isolines, pluviograph pluviometer.

\section{INTRODUCCIÓN}

La red hidrometeorológica colombiana está conformada por estaciones de distintas categorías de instituciones para el control, monitoreo y manejo ambiental, entre las cuales se destaca el Ideam como la mayor institución suministradora de información. Estas cuentan con información hidrológica fundamental para su aplicación dentro de los estudios básicos y diseños para la construcción de obras civiles; dado que el factor hidrológico es fundamental para considerar dentro de la operación de este tipo de obras, es necesario contar con información actualizada de este tipo con cubrimiento del área específica en estudio. No obstante, para el caso del territorio colombiano, 
se presenta un déficit en cuanto a la disponibilidad y procesamiento de información hidrológica. El principal problema radica en que el número de estaciones hidrometeorológicas no cubre convenientemente la extensión territorial del país, puesto que según las indicaciones de la Organización Meteorológica Mundial, una estación cubre adecuadamente un territorio comprendido por un máximo de 15 kilómetros; sin embargo, por lo general las estaciones se encuentran separadas por una longitud mayor, con lo que se dejan grandes extensiones sin cubrimiento. A esto se suma que la información registrada no se encuentra apropiadamente procesada, lo cual requiere un extenso y exhaustivo procedimiento estadístico.

Mediante esta investigación se desarrolla y aplica una metodología para regionalizar curvas IDF mediante el uso de registros pluviográficos, de tal forma que se permita obtener las constantes que describen las relaciones entre las variables de la precipitación: intensidad, duración y frecuencia, a partir de una coordenada de localización del punto de estudio ubicado dentro del departamento.

Para el caso de esta investigación, se ha tomado como zona de estudio el departamento de Boyacá, aprovechando que se trata de una región con gran diversidad de climas gracias a su variedad de altitudes que pueden ir desde los 300 hasta los 3.000 m s.n.m., además, por ser uno de los departamentos que cuenta con un buen número y una buena distribución de estaciones hidrometeorológicas en la extensión de su territorio.

La regionalización de curvas IDF ya ha sido anteriormente estudiada en el país, mediante diversas metodologías ya desarrolladas para otros países. Se destaca como investigación más importante la adelantada por Díaz-Granados y Vargas (1997), con la cual se logró regionalizar la totalidad del territorio colombiano, generalizando cuatro zonas mediante ecuaciones generales. Está regionalización es aceptada y utilizada en el país, para el cálculo de curvas IDF usadas en proyectos típicos de ingeniería que requieran dicha información.

\section{METODOLOGÍA}

\section{Determinación del área de estudio}

Antes de iniciar y proyectar la investigación se establece un área de estudio que asegure un mínimo de condiciones para poder llevar a cabo un adecuado estudio, y que a su vez esta área tenga un límite máximo en cuanto a su extensión, de tal forma que sea posible desarrollar apropiadamente el estudio partiendo de la limitante del tiempo disponible y el personal incurso en el proyecto.

Dado lo anterior, se inicia este estudio, proyectado en un futuro a cubrir todo el territorio nacional colombiano, limitando la investigación al área comprendida por el departamento de Boyacá. Posteriormente, con el área de estudio definida, se procede a seleccionar un determinado número de estaciones hidrometeorológicas, que cumplan con los requisitos mínimos requeridos por este proyecto, y que a su vez estén distribuidas de manera homogénea dentro del departamento.

Cumpliendo las condiciones anteriormente definidas, se determina un número total de 20 estaciones hidrometeorológicas que, según categorización del Ideam, cuentan al menos con pluviómetro y pluviógrafo.

En la tabla 1 se relacionan las estaciones contempladas dentro de la investigación, según catalogación de Ideam; y en la figura 1, se ilustra el plano de localización de dichas estaciones dentro del departamento de Boyacá.

\section{Recolección y procesamiento de información}

Desarrollar esta investigación requiere una base de información previa, comprendida por un compilado de hidrometerología correspondiente al conjunto de las 20 estaciones mínimo de categoría PG, según clasificación de Ideam (Ardila, 1997). Con esta información se procede a calcular las curvas IDF para todas las estaciones, con el fin de obtener los parámetros $K, d_{0^{\prime}} m$ y $n$, que serían el objeto de regionalización (Desramaut, 2008). 
Tabla 1. Estaciones meteorológicas región Boyacá incluidas en estudio

\begin{tabular}{ccccc}
\hline \multicolumn{5}{c}{ Información de la estación } \\
\hline No. & Código & Nombre y código & Municipio & Corriente \\
\hline 1 & 35070260 & ALMEIDA [35070260] & ALMEIDA & BATA \\
\hline 2 & 24035340 & APTO A LLERAS C [24035340] & SOGAMOSO & CHICAMOCHA \\
\hline 3 & 23125140 & APTO FURATENA [23125140] & QUIPAMA & MINERO \\
\hline 4 & 24030820 & AZULEJOS LOS [24030820] & TUTA & TUTA \\
\hline 5 & 24035150 & BELENCITO [24035150] & NOBSA & CHICAMOCHA \\
\hline 6 & 24035040 & COPA LA [24035040] & TOCA & TUTA \\
\hline 7 & 35070080 & GARAGOA [35070080] & GARAGOA & GARAGOA \\
\hline 8 & 35070110 & GUAYATA LA GRANJA [35070110] & GUICÁN & SOMONDOCO \\
\hline 9 & 24035070 & GUICAN [24035070] & OTANCHEVADO & QDA. TAMBRIAS \\
\hline 10 & 23125080 & OTANCHE [23125080] & PÁEZ & MUECHE \\
\hline 11 & 35085030 & PAEZ [35085030] & OLARTE \\
\hline 12 & 35090010 & POTRERITO [35090010] & CHIQUIZA & MAGDALENA \\
\hline 13 & 23115010 & PTO. BOYACÁ [23115010] & CHULO \\
\hline 14 & 24010830 & SAN PEDRO IGUAQUE [24010830] & SUTIVANORTE & QDA. LAS LEONAS \\
\hline 15 & 24035320 & SATIVANORTE [24035320] & PAIPA & GARAGOA \\
\hline 16 & 35075020 & SUTATENZA [35075020] & SAMACÁ & SALITRE \\
\hline 17 & 24035170 & TUNGUAVITA [24035170] & RAMIRIQUÍ & TEATINOS \\
\hline 18 & 24015220 & VILLA CARMEN [24015220] & MIRAFLORES & LENGUPA \\
\hline 19 & 35075030 & VILLA LUISA [35075030] & &
\end{tabular}

Fuente: elaboración propia.

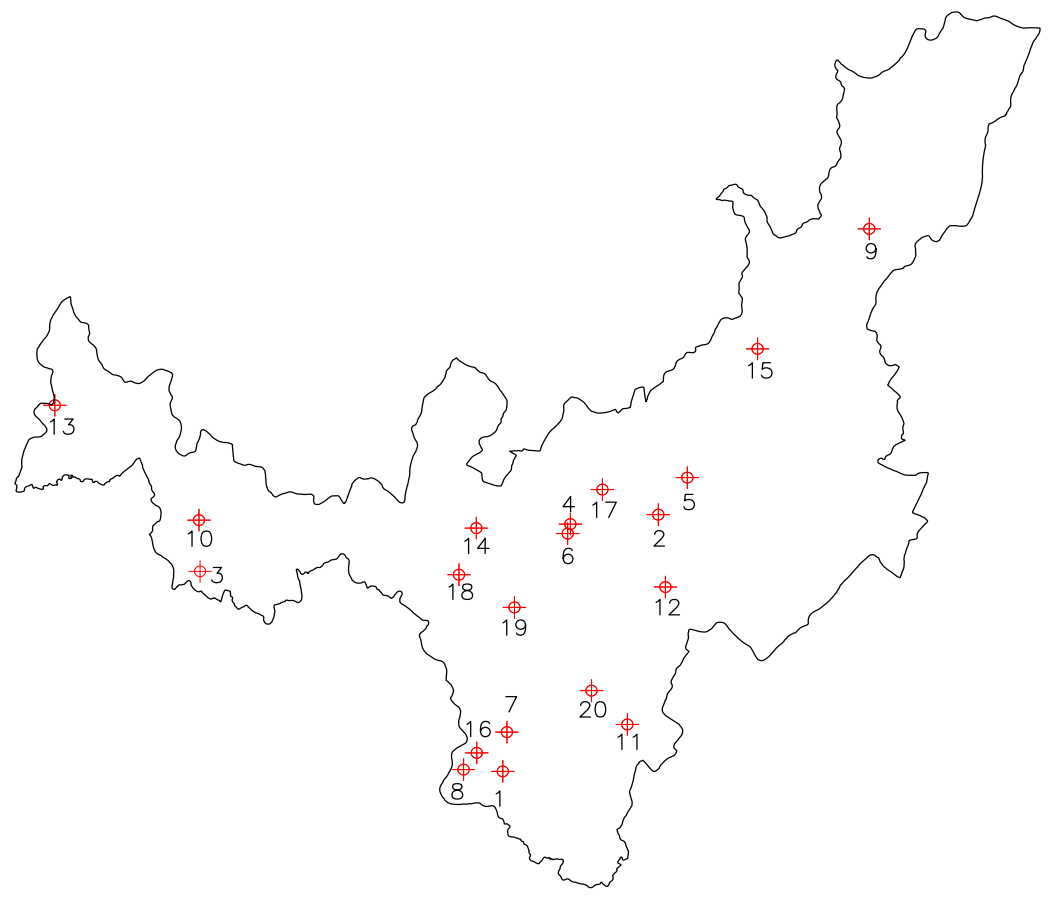

Figura 1. Localización de estaciones meteorológicas dentro del departamento

Fuente: elaboración propia. 
En la tabla 2 se presenta un ejemplo de la información primaria empleada para el cálculo de las curvas IDF; en ella se presenta la evaluación de las bandas pluviográficas de la estación Páez (Boyacá) en intervalos de 10, 20, 30, 60, 120, 180, 240 y 360 minutos.

A partir de la información presentada en la tabla 2, se procede a calcular la intensidad del evento multianual relacionado a cada intervalo de duración, mediante la ecuación (1) (Chow, 1994). El resultado de este proceso se presenta en la tabla 3.

$$
i=\frac{P(\operatorname{mm})}{d(\min )} \times\left(\frac{60 \mathrm{~min}}{1 \mathrm{~h}}\right)=\left[\frac{\mathrm{mm}}{\mathrm{h}}\right]
$$

Tabla 2. Resultados de evaluación de bandas pluviográficas, estación Páez (Boyacá)

\begin{tabular}{ccccccccc}
\hline & \multicolumn{7}{c}{$\mathbf{P}(\mathbf{m m}) \mathbf{m i n}$} \\
\hline AÑ̃ & $\mathbf{1 0}$ & $\mathbf{2 0}$ & $\mathbf{3 0}$ & $\mathbf{6 0}$ & $\mathbf{1 2 0}$ & $\mathbf{1 8 0}$ & $\mathbf{2 4 0}$ & $\mathbf{3 6 0}$ \\
\hline 1992 & 7,8 & 9,7 & 12,2 & 15,4 & 17,2 & 16,4 & 18,0 & 19,9 \\
\hline 1993 & 4,0 & 7,7 & 10,7 & 14,1 & 14,7 & 16,6 & 16,9 & 17,4 \\
\hline 1994 & 10,3 & 15,4 & 20,5 & 25,8 & 26,7 & 26,9 & 26,9 & 33,4 \\
\hline 1995 & 6,2 & 9,5 & 10,9 & 15,1 & 17,6 & 20,0 & 20,1 & 14,3 \\
\hline 1996 & 8,6 & 12,0 & 17,9 & 26,5 & 29,4 & 31,0 & 31,6 & 31,7 \\
\hline 1997 & 7,5 & 14,3 & 16,1 & 20,8 & 23,8 & 25,1 & 25,5 & 25,7 \\
\hline 1998 & 9,6 & 16,7 & 25,1 & 25,2 & 25,2 & 25,2 & 25,2 & 25,2 \\
\hline 1999 & 4,2 & 7,4 & 8,1 & 11,4 & 15,1 & 15,5 & 15,9 & 17,4 \\
\hline 2000 & 5,7 & 8,9 & 10,4 & 15,4 & 17,1 & 20,3 & 23,4 & 26,7 \\
\hline 2001 & 3,7 & 6,6 & 8,1 & 9,3 & 10,8 & 11,0 & 11,0 & 15,2 \\
\hline 2002 & 8,5 & 16,5 & 18,9 & 22,4 & 25,8 & 27,6 & 28,8 & 28,9 \\
\hline 2003 & 2,5 & 4,4 & 5,7 & 7,1 & 10,5 & 13,1 & 16,3 & 21,5 \\
\hline 2004 & 6,0 & 9,4 & 12,5 & 17,7 & 22,9 & 25,8 & 25,8 & 25,8 \\
\hline 2005 & 2,1 & 3,5 & 4,4 & 4,8 & 7,6 & 10,1 & 11,6 & 12,5 \\
\hline 2006 & 6,8 & 12,6 & 17,3 & 24,5 & 29,7 & 29,7 & 29,7 & 29,7 \\
\hline
\end{tabular}

Fuente: elaboración propia.

Tabla 3. Valores multianuales de intensidad presentados para cada intervalo de duración

\begin{tabular}{|c|c|c|c|c|c|c|c|c|}
\hline \multirow[b]{2}{*}{ AÑO } & \multicolumn{8}{|c|}{$P(\mathrm{~mm} / \mathrm{h})$} \\
\hline & 10 & 20 & 30 & 60 & 120 & 180 & 240 & 360 \\
\hline 1992 & 46,8 & 29,1 & 24,4 & 15,4 & 8,6 & 5,5 & 4,5 & 3,3 \\
\hline 1993 & 24,0 & 23,1 & 21,4 & 14,1 & 7,4 & 5,5 & 4,2 & 2,9 \\
\hline 1994 & 61,8 & 46,2 & 41,0 & 25,8 & 13,4 & 9,0 & 6,7 & 5,6 \\
\hline 1995 & 37,2 & 28,5 & 21,8 & 15,1 & 8,8 & 6,7 & 5,0 & 2,4 \\
\hline 1996 & 51,6 & 36,0 & 35,8 & 26,5 & 14,7 & 10,3 & 7,9 & 5,3 \\
\hline 1997 & 45,0 & 42,9 & 32,2 & 20,8 & 11,9 & 8,4 & 6,4 & 4,3 \\
\hline 1998 & 57,6 & 50,1 & 50,2 & 25,2 & 12,6 & 8,4 & 6,3 & 4,2 \\
\hline 1999 & 25,2 & 22,2 & 16,2 & 11,4 & 7,6 & 5,2 & 4,0 & 2,9 \\
\hline 2000 & 34,2 & 26,7 & 20,8 & 15,4 & 8,6 & 6,8 & 5,9 & 4,5 \\
\hline 2001 & 22,2 & 19,8 & 16,2 & 9,3 & 5,4 & 3,7 & 2,8 & 2,5 \\
\hline 2002 & 51,0 & 49,5 & 37,8 & 22,4 & 12,9 & 9,2 & 7,2 & 4,8 \\
\hline 2003 & 15,0 & 13,2 & 11,4 & 7,1 & 5,3 & 4,4 & 4,1 & 3,6 \\
\hline 2004 & 36,0 & 28,2 & 25,0 & 17,7 & 11,5 & 8,6 & 6,5 & 4,3 \\
\hline 2005 & 12,6 & 10,5 & 8,8 & 4,8 & 3,8 & 3,4 & 2,9 & 2,1 \\
\hline 2006 & 40,8 & 37,8 & 34,6 & 24,5 & 14,9 & 9,9 & 7,4 & 5,0 \\
\hline
\end{tabular}

Fuente: elaboración propia. 
Una vez, habiendo calculado estas series de intensidad máxima multianual, se procede a aplicar cuatro de las funciones de distribución de probabilidad típicas usadas en hidrología como lo sugiere Aparicio (1999): normal, log-normal, Pearson III y Gumbel; de las cuales se encoge una sola para ajustar la información de las 20 estaciones analizadas mediante el uso de las pruebas de bondad de ajuste: Kolmogorov-Smirnov, error cuadrático mínimo y chi-Cuadrado (Canavos, 1995). Tras la aplicación de estas cuatro funciones de distribución de probabilidad y las correspondientes pruebas de bondad de ajuste se determina que la función que mejor ajusta la mayoría de la información es Gumbel, por lo cual para los procesos de regionalización se ajusta toda la información únicamente a esta función, con el fin de lograr homogeneidad entre las variables que relacionan la intensidad, la duración y el periodo de retorno para todas las estaciones, dado que las diferencias entre modelos probabilísticos son significativas. Esto generaría conflictos al aplicar los métodos de regionalización. En la tabla 4 se relacionan las variables obtenidas para las 20 estaciones analizadas $\left(K, d_{0^{\prime}} m\right.$ y $n)$.

Estas cuatro variables se interrelacionan mediante la ecuación (2) (Monsalve, 1995), que permite calcular la intensidad que puede llegar a presentarse durante cierta duración y a determinado periodo de retorno (Pizarro, 1996). En la tabla 5 se presenta, para una de las estaciones trabajadas, la estimación de la intensidad de las precipitaciones relacionadas con periodos de retorno y tiempos de precipitación comúnmente utilizados.

Tabla 4. Constantes de ecuaciones IDF para estaciones estudiadas

\section{CONSTANTES DE ECUACIONES IDF}

\begin{tabular}{|c|c|c|c|c|}
\hline Nombre y código de la estación & k & $\mathbf{m}$ & to & $\mathbf{n}$ \\
\hline ALMEIDA [35070260] & 2312,7 & 0,1532 & 53,77 & 1 \\
\hline APTO A LLERAS C [24035340] & 1603,9 & 0,1723 & 18,64 & 1 \\
\hline APTO FURATENA [23125140] & 1832,0 & 0,1612 & 16,75 & 1 \\
\hline AZULEJOS LOS [24030820] & 1741,1 & 0,1858 & 17,15 & 1 \\
\hline BELENCITO [24035150] & 1739,3 & 0,1677 & 24,10 & 1 \\
\hline COPA LA [24035040] & 1463,7 & 0,1775 & 21,05 & 1 \\
\hline GARAGOA [35070080] & 2020,8 & 0,1372 & 46,11 & 1 \\
\hline GUAYATÁ LA GRANJA [35070110] & 1624,1 & 0,1909 & 40,91 & 1 \\
\hline GUICÁN [24035070] & 1442,8 & 0,2180 & 23,91 & 1 \\
\hline OTANCHE [23125080] & 5758,6 & 0,1702 & 41,35 & 1 \\
\hline PÁEZ [35085030] & 2289,1 & 0,1873 & 32,93 & 1 \\
\hline POTRERITO [35090010] & 1417,5 & 0,1851 & 34,78 & 1 \\
\hline PTO BOYACÁ [23115010] & 5054,6 & 0,1655 & 42,8 & 1 \\
\hline SAN PEDRO IGUAQUE [24010830] & 1616,1 & 0,1550 & 28,21 & 1 \\
\hline SATIVANORTE [24035320] & 2217,9 & 0,1986 & 25,20 & 1 \\
\hline SUTATENZA [35075020] & 1705,6 & 0,1694 & 25,36 & 1 \\
\hline TUNGUAVITA [24035170] & 1922,7 & 0,2083 & 19,70 & 1 \\
\hline VILLA CARMEN [24015220] & 2142,0 & 0,1938 & 22,96 & 1 \\
\hline VILLA LUISA [35075030] & 1526,7 & 0,1732 & 10,63 & 1 \\
\hline VIVERO EL [35085040] & 2346,4 & 0,1878 & 49,22 & 1 \\
\hline
\end{tabular}

Fuente: elaboración propia. 
Tabla 5. Evaluación ecuación IDF, estación Páez (Boyacá)

\begin{tabular}{ccccccc}
\hline \multicolumn{7}{c}{ Evaluación ecuación IDF, estación Páez (Boyacá) } \\
\hline $\begin{array}{c}\text { Duración } \\
(\mathbf{m i n})\end{array}$ & $\mathbf{5}$ & $\mathbf{5}$ & $\mathbf{1 0}$ & $\mathbf{2 5}$ & $\mathbf{5 0}$ & $\mathbf{1 0 0}$ \\
\cline { 2 - 7 } & 55,1 & 65,3 & 82,3 & 111,7 & 140,7 & 177,2 \\
\hline 5 & 49,0 & 58,0 & 73,1 & 99,2 & 125,0 & 157,5 \\
\hline 10 & 44,1 & 52,2 & 65,8 & 89,3 & 112,5 & 141,7 \\
\hline 15 & 40,1 & 47,5 & 59,8 & 81,2 & 102,3 & 128,8 \\
\hline 20 & 33,9 & 40,2 & 50,6 & 68,7 & 86,6 & 109,0 \\
\hline 30 & 23,2 & 27,5 & 34,7 & 47,1 & 59,3 & 74,7 \\
\hline 60 & 14,3 & 16,9 & 21,3 & 28,9 & 36,4 & 45,9 \\
\hline 120 & 10,3 & 12,2 & 15,4 & 20,9 & 26,3 & 33,2 \\
\hline 180 & 8,1 & 9,6 & 12,1 & 16,4 & 20,6 & 26,0 \\
\hline 240 & 5,6 & 6,7 & 8,4 & 11,4 & 14,4 & 18,1 \\
\hline 360 & & & & &
\end{tabular}

Fuente: elaboración propia.

Graficando cada una de las series obtenidas, descritas por un mismo periodo de retorno, se obtienen las curvas IDF, como el ejemplo presentado en la figura 2.

$$
i=\frac{K \cdot T^{m}}{\left(d+d_{0}\right)^{n}}
$$

Donde:

$i$ : intensidad de precipitación máxima, en mm/ hora.

$d$ : duración de la lluvia, en minutos.

$d_{0}$ : constante, en minutos.

$K, m, y n$ : constantes adimensionales.

La constante se determina mediante un procedimiento de ensayo y error con el cual se logra un ajuste óptimo de las series, a un modelo de regresión potencial, en donde la mayor aproximación de dicha constante se refleja en un coeficiente de determinación, cercano a 1 . Esto conlleva el importante beneficio de que la constante tiende a 1,0. Asi, la nueva ecuación, para relacionar las variables IDF, queda como se presenta a continuación (ecuación 3).

Dado lo anterior, se observa que la ecuación se reduce a solo tres constantes. Por consiguiente, el proceso de regionalización considera únicamente las constantes: $K, d_{0}$ y $m$, simplificando de esta manera el procedimiento.

\section{Procedimiento de regionalización}

Para elegir el método de regionalización más adecuado y aplicable en el caso de la muestra ensayada, se realiza una evaluación general de las constantes que se desean regionalizar (Tapia, 2001). De esta evaluación se observa que las magnitudes de las constantes tienden a ser cercanas unas de otras, encerradas dentro de un rango pequeño. Por tanto, usar el método de mapa de isolíneas sería adecuado para efectuar la regionalización (Stanescu, 1994).

Para desarrollar la regionalización se aplican procedimientos matemáticos y geométricos senciIlos, que permiten triangular e interpolar desde los puntos existentes, hasta lograr un mapa completo con isolíneas; uno para cada una de las tres variables analizadas, $K, m$ y $d_{0}$.

El primer paso es realizar una triangulación entre los puntos representados por la ubicación geográfica de cada estación para facilitar de esta 


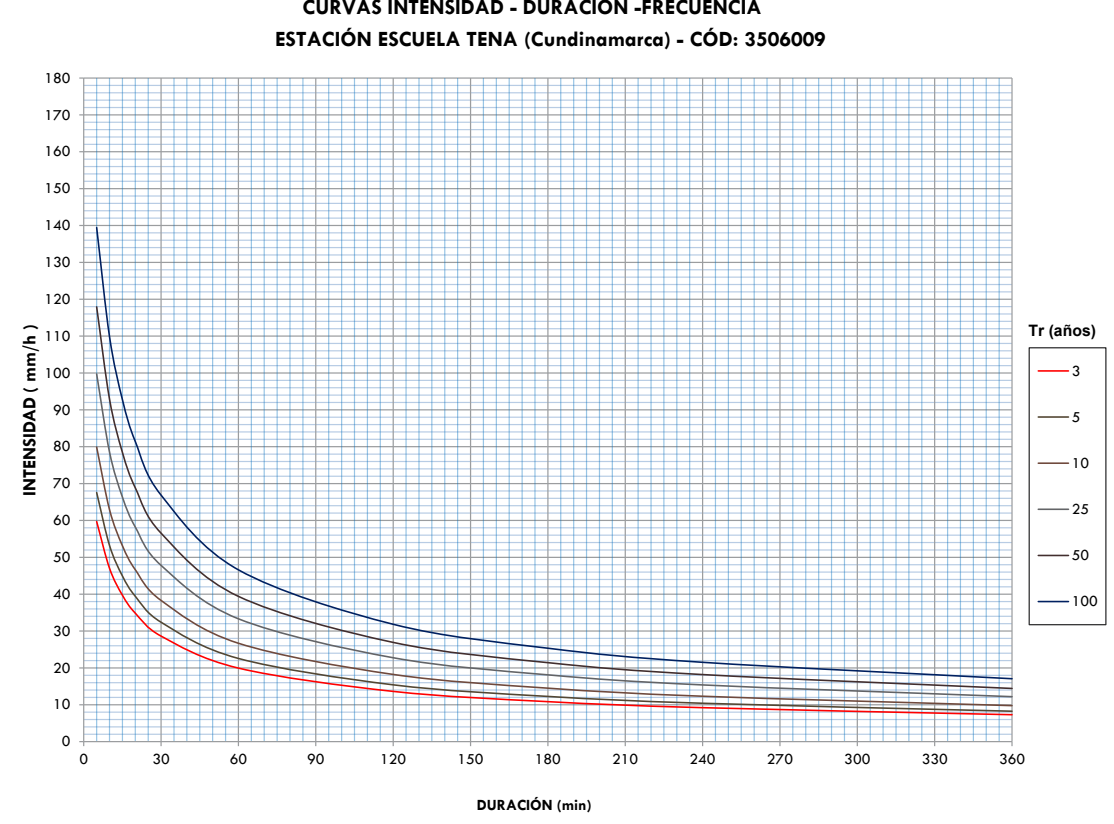

Figura 2. Ejemplo de curva IDF

Fuente: Elaboración propia.

manera el proceso de interpolación. Este paso se representa en la figura 3.

Antes de realizar la interpolación, se asigna como coordenada X, Y y Z, la longitud geográfica, la latitud geográfica y el valor de la constante $(K$, $m$ y $d_{0^{\prime}}$ según corresponda), respectivamente, para cada una de las estaciones consideradas.

Los resultados de estas interpolaciones se presentan en las figuras 4, 5 y 6 , como planos de isolíneas las constantes $m, K$ y $t_{0^{\prime}}$ respectivamente.

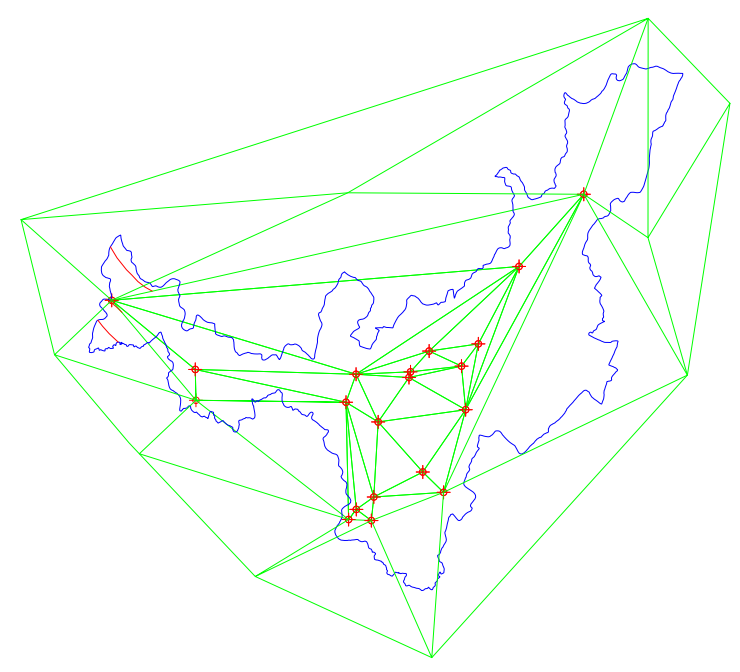

Figura 3. Triangulación entre coordenadas de referencia

Fuente: elaboración propia. 


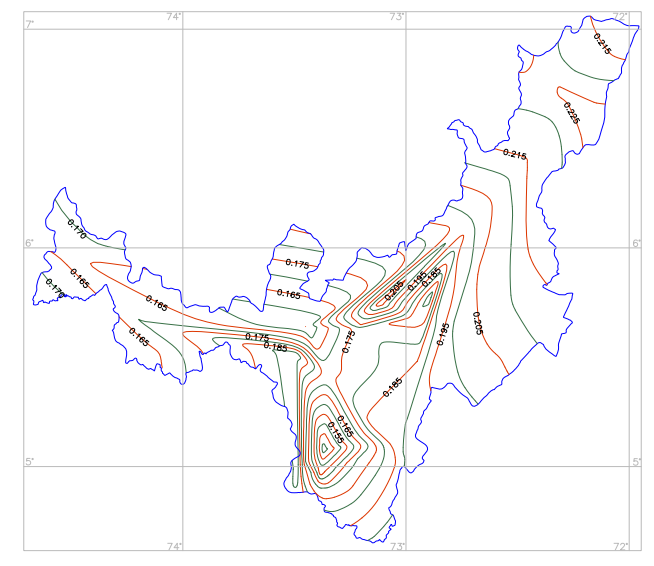

Figura 4. Isolíneas de regionalización constante $m$

Fuente: elaboración propia.

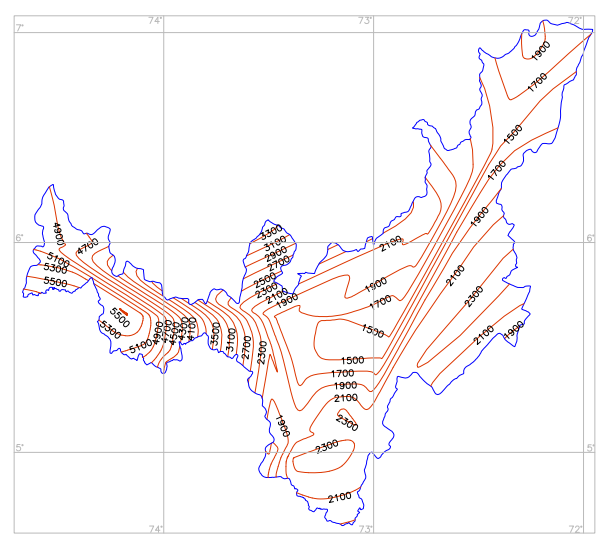

Figura 5. Isolíneas de regionalización constante $K$

Fuente: elaboración propia.

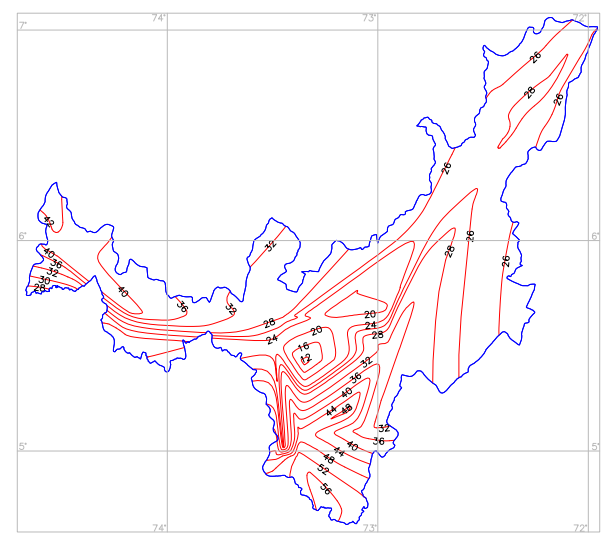

Figura 6. Isolíneas de regionalización constante $t_{o}$

Fuente: elaboración propia. 
A partir de los mapas de las figuras 4,5 y 6 , es posible determinar cada una de las constantes que definen la ecuación de las curvas IDF, ubicando la coordenada geográfica correspondiente al punto sobre el cual se va a trabajar.

\section{RESULTADOS}

Para demostrar la validez de los resultados, representados por los planos de isolíneas generados mediante esta investigación, se toma como referencia dos estaciones no contempladas dentro de estudio, de las cuales se conoce las constantes de regionalización $\left(K, m, n\right.$ y $\left.d_{0}\right)$ de tal manera que se comparen los valores teóricos calculados mediante el procedimiento convencional frente a los obtenidos mediante los mapas de isolíneas ubicando la estación considerada para el análisis.

En la tabla 6, se presentan los datos de las estaciones consideradas para esta comprobación junto a los respectivos parámetros para realizar la verificación.

En las figuras 7, 8 y 9 se observa la localización de las estaciones de prueba, dentro de cada uno de los planos de isolíneas, de los cuales se extrae el valor correspondiente para cada constante regionalizada: $K, m$ y $d_{0}$, respectivamente.

Tabla 6. Parámetros ecuación IDF estaciones de prueba

\begin{tabular}{lcccc}
\hline \multicolumn{5}{c}{ Constantes de ecuaciones IDF } \\
\hline \multicolumn{1}{c}{ Nombre y código de la estación } & $\mathbf{k}$ & $\mathbf{~ m}$ & to & $\mathbf{n}$ \\
\hline CHISCAS [24035310] & 1825,9 & 0,2388 & 29,61 & 1 \\
\hline CAMPO HERMOSO [35085050] & 2535,4 & 0,1924 & 45,38 & 1 \\
\hline
\end{tabular}

Fuente: elaboración propia.

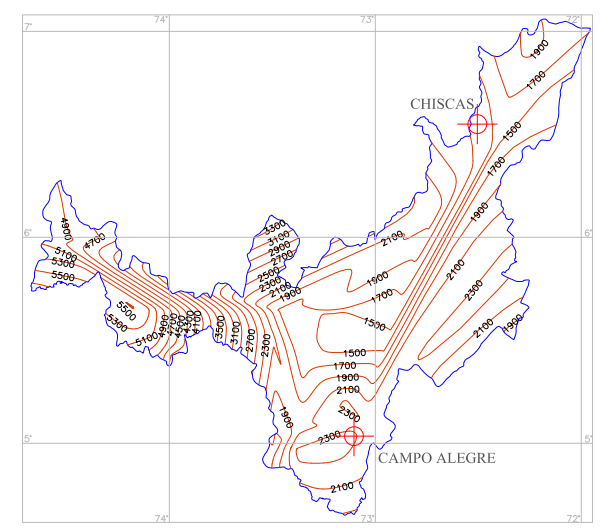

Figura 7. Localización de estaciones de prueba en plano de Isolíneas de regionalización constante $k$

Fuente: elaboración propia.

Para deducir el valor de las constantes que componen la ecuación IDF, para las estaciones tomadas como prueba, se parte de que el valor para la constante $n$ tiene un valor de 1, acorde con el proceso de regionalización. Tras la localización de las estaciones dentro de cada uno de los planos de isolíneas, se logra determinar el valor de las constantes regionalizadas $\left(K, m\right.$ y $\left.d_{0}\right)$ mediante procesos de interpolación entre las isolíneas. En la tabla 7 se presentar el valor de las contantes de la ecuación IDF, que describe el comportamiento de las precipitaciones en la zona cubierta por las estaciones de prueba. 


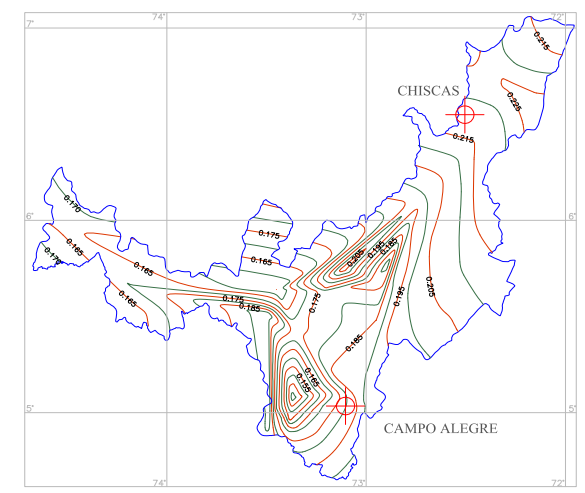

Figura 8. Localización de estaciones de prueba en plano de isolíneas de regionalización constante $m$ Fuente: elaboración propia.

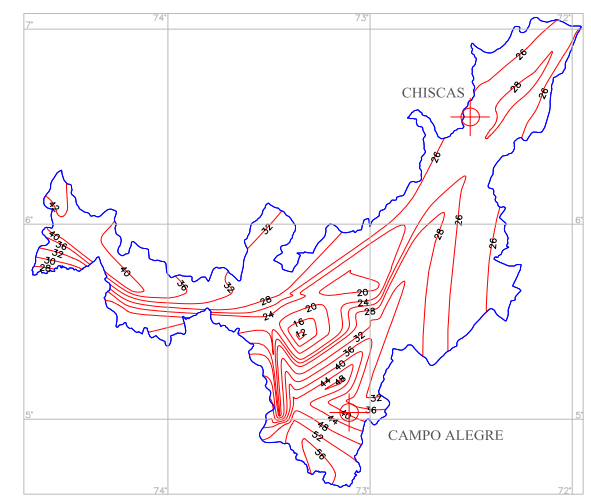

Figura 9. Localización de estaciones de prueba en plano de isolíneas de regionalización constante $t_{o}$

Fuente: elaboración propia.

Tabla 7. Parámetros ecuación IDF estaciones de prueba

\begin{tabular}{lccccc}
\hline \multicolumn{4}{c}{ Constantes de ecuaciones IDF } & & \\
\hline \multicolumn{1}{c}{ Nombre y código de la estación } & $\mathbf{k}$ & $\mathbf{~ m}$ & to & $\mathbf{n}$ \\
\hline CHISCAS [24035310] & 1650,5 & 0,2180 & 26,6 & 1 \\
\hline CAMPO HERMOSO [35085050] & 2343,5 & 0,1790 & 41,2 & 1 \\
\hline
\end{tabular}

Fuente: elaboración propia.

Una vez extraídos estos valores para las constantes, se comparan a los calculados paso a paso mediante el método convencional presentado al inicio de este artículo, con el fin evaluar el error presentado, y su validez para su uso. En la tabla 8 se muestra el error presentado en la extracción de cada una de las constantes regionalizadas.
Considerando la precisión de estos planos de isolíneas y el funcionamiento del método de regionalización, el error presentado es aceptable dentro de los términos de confiabilidad para su aplicación como información válida que describa las relaciones de intensidad, duración y frecuencia de las precipitaciones en la zona comprendida por 
Tabla 8. Error presentado en parámetros regionalizados

\begin{tabular}{lcccc}
\hline \multicolumn{4}{c}{ Error en constantes regionalizadas (\%) } \\
\hline \multirow{2}{*}{ Nombre y código de la estación } & \multicolumn{4}{c}{ Constante } \\
\cline { 2 - 5 } & $\mathbf{k}$ & $\mathbf{~ m}$ & to & $\mathbf{n}$ \\
\hline CHISCAS [24035310] & 10,63 & 9,56 & 11,32 & 1 \\
\hline CAMPO HERMOSO [35085050] & 8,19 & 7,48 & 10,14 & 1 \\
\hline
\end{tabular}

Fuente: elaboración propia.

esta investigación que contempla el territorio del departamento de Boyacá.

\section{CONCLUSIONES}

El método de isolíneas demuestra ser aplicable para la regionalización de variables de ecuaciones que representen modelos hidrológicos.

Regionalizar ecuaciones para el cálculo de curvas IDF, mediante el uso de isolíneas para cada variable, representa el método más adecuado para el usuario dado que le permite determinar con una gran precisión las variables que componen dicha ecuación $\left(k, m\right.$ y $\left.d_{0}\right)$, sin necesidad de evaluar información registrada por pluviómetro o pluviógrafo.

Las herramientas estocásticas son fundamentales dentro de los procesos de regionalización dado que, independientemente del método de regionalización empleado, se analiza, procesa y compara información correspondiente a series multianuales que describen patrones estadísticos y probabilísticos que pueden ser descritos mediante modelos matemáticos.

\section{AGRADECIMIENTOS}

Los autores agradecen al director del Ideam, Omar Franco Torres, por aprobar favorablemente el desarrollo de este proyecto en el marco del convenio interinstitucional 002 de 2008 suscrito entre estas dos instituciones; al coordinador del Grupo Archivo Técnico, Nelson Arlington Castro Beltrán, por suministrar abiertamente toda la información necesaria para adelantar este estudio científico, y a la funcionaria
Amparo Murcia Suárez, por su importante respaldo en la compilación y transmisión de la información; así mismo, agradecen a la Universidad Distrital Francisco José de Caldas y al Semillero de Investigación Udens por la promoción de la investigación.

\section{REFERENCIAS}

Aparicio M., F.J. (1999). Fundamentos de hidrología de superficie. México D.F.: Limusa.

Ardila H., G.A. (1997). Guía de construcciones hidrometeorológicas. Bogotá: Ideam.

Canavos, G. (1995). Probabilidad y estadística. Aplicaciones y métodos. México: McGraw-Hill Interamericana.

Chow, V.T. (1994). Hidrología aplicada. Bogotá: McGraw-Hill.

Desramaut, N. (2008). Estimation of Intensity Duration Frequency Curves for Curren and Future Climates. Montreal: McGill University.

Díaz-Granados O., M. y Vargas M., R. (1997). Curvas sintéticas regionalizadas de intensidad-duración-frecuencia para Colombia. Bogotá: Universidad de los Andes.

Monsalve S., G. (1995). Hidrología en la ingeniería. Bogotá: Escuela Colombiana de Ingeniería.

Pizarro T., R. (1996). Curvas intensidad duración frecuencia para la zona centro sur de Chile. Chile: Programa Hidrológico Internacional de la UNESCO.

Stanescu, V.A. (1994). Procedures for hydrological regionalization applied in Romania. Rumania: National Institute of Meteorology and Hydrology.

Tapia, R.P. (2001). Análisis comparativo de las curvas intensidad - duración -frecuencia (IDF) en 6 estaciones pluviográficas (VII Región del Maule, Chile). Chile: Universidad de Talca.

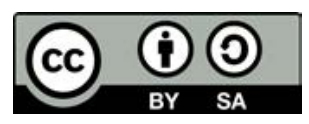

Tecnura • p-ISSN: 0123-921X • e-ISSN: 2248-7638 • Vol. 22 No. 58・ Octubre - Diciembre de $2018 \bullet$ pp. 53-64 